\title{
Granular Matter: a wonderful world of clusters in far-from-equilibrium systems
}

\author{
M. Ausloos ${ }^{a}$, R. Lambiotte ${ }^{\mathrm{a}, \mathrm{b}}, \mathrm{K}$. Trojan $^{\mathrm{a}, \mathrm{c}}, \mathrm{Z}$ Koza $^{\mathrm{a}, \mathrm{c}}$, \\ M. Pekala ${ }^{a, d}$ \\ a SUPRATECS, B5, Sart Tilman Campus, B-4000 Liège, Euroland \\ ${ }^{\mathrm{b}}$ Université Libre de Bruxelles, B-1000 Bruxelles, Euroland \\ ${ }^{\mathrm{c}}$ Institute of Theoretical Physics, University of Wroctaw, pl. Maxa Borna 9, \\ PL-50-204 Wroctaw, Poland \\ ${ }^{\mathrm{d}}$ Department of Chemistry, University of Warsaw, \\ Al. Żwirki i Wigury 101, PL-02-089 Warsaw, Poland
}

\begin{abstract}
In this paper, we recall various features of non equilibrium granular systems. Clusters with specific properties are found depending on the packing density, going from loose (a granular gas) to sintered (though brittle) polycrystalline materials. The phase space available can be quite different. Unexpected features, with respect to standard or expected ones in classical fluids or solids, are observed, - like slow relaxation processes or anomalous electrical and thermoelectrical transport property dependences. The cases of various pile structures and the interplay between classical phase transitions and self-organized criticality for avalanches are also outlined.
\end{abstract}

Key words: Granular models of complex systems, Self-organized systems PACS: 89.65.Gh, 05.10.Ln, 89.75.-k, 07.05.Tp, 05.65.+b

\section{Introduction}

Granular media are made of a large number of macroscopic solid entities. The size of these particles ranges from that of dust (in powders) to that of rocks (in planetary rings). Practically let us note that grains often result from fracture processes rather than from physico-chemical synthesis. The granular media well-known features encompass avalanches and agglomerations, By extension

Email address: MarcelAusloos@ulg.ac.be (M. Ausloos). 
of the statistical physics point of view, many other systems can be considered as granular systems, for example car traffic is thought to be similar to granular flow.

Transport and storage of granular materials may also be problematic, and have striking consequences, as in the case of the storage of cereals in silos that may break due to the huge internal pressure forces generated by the grains. In the pharmaceutical industry, the properties of mixing and segregation of these systems is primordial to optimize the contact area between the chemical components, and consequently the efficiency of the chemical reaction; moreover, in the food industry, the properties of homogenization go on par with the standardization required by mass production. For these reasons, in the engineering community, there has been a long-standing interest in describing and predicting both static and dynamical properties of granular materials.

It is thought that the properties of granular materials mainly originate from their macroscopic dimensions and surface interactions between the grains, which at the relevant scale of granular physics are considered as solid objects whose internal degrees of freedom can be neglected. This has important consequences: the ordinary temperature plays no role on the grain motion, and the interaction between grains is usually dissipative. Yet, whether the granules in such materials interact through attractive or repulsive forces is not a trivial question. This is somewhat unusual physics, and should have attracted physicist attention.

All this points to a very rich phenomenology which in many cases qualitatively differs from that observed in the familiar forms of matter, namely solids, liquids or gases. Over the last decades, their striking features have been put in parallel with phenomena occurring in more complex systems, and have been used as a fruitful metaphor to describe other dissipative dynamical systems. For instance, sand piles have been used by De Gennes (1) as a macroscopic picture for the motion of flux lines in superconductors, and by Bak (2) as a paradigm of the Self-Organized Criticality (SOC) concept. Other fields of science have borrowed ideas from granular matter physics, such as crystal agglomeration and sintering, traffic (jams), film growth, galaxy clustering, cloud formation (aerosols), company merging (city planning), fracture. The slow relaxation processes which take place in vibrated sand piles as well as in glasses or flux lattices $(3 ;$; $)$ are other examples. Macroscopic properties, i.e. through mechanical, thermal, electric or magnetic measurements, can be used to test the internal structure of piles or compacted matter. Their interpretation remains a fundamental challenge. Nevertheless it can be expected that macroscopic truly natural systems will be described and explained from microscopic models. This attempt is hereby made under the form of a review of work by various of us. 
In Sect. 2, a few results on Granular Gases will be presented, though for a more complete presentation, it is best to read the paper by Lipowski (5) in these proceedings, and the thesis by Lambiotte (6). The Maxwell Demon experiment will be recalled and it will be shown that granular gases present complex features such as clustering, metastability, segregation... The fact that the velocities of the grains do not distribute themselves like a Maxwellian will also be shortly presented. Non-equipartition of energy in mixtures and non ergodicity will not be discussed for lack of space. The Ballistic Magnetic Deposition (BMD) model (7) of granular piles, somewhat related to the Tetris model (8) will be discussed in Sect.3. The role of contact energy, bead anisotropy, and external field influence will be mentioned in order to warn about problems which might arise for the stress net estimation and the formation of clusters. The Bak-Tang-Wiesenfeld (BTW) Sand Pile model (9) will find its place in Sect. 4, where it will be coupled to the Blume-Emery-Grifiths (BEG) model (10) in order to test the robustness of SOC. The observation of log-periodic avalanches in piles built on a fractal basis will be recalled to have led to a model for the analogous behavior of log-periodic oscillations in financial indices before an endogenous crash. An anomalous behavior of the electrical resistivity and the thermoelectric power (TEP) will be recalled in Sect. 5 for densely packed conducting grains. Conclusions can be found in Sect. 6

\section{Granular Gases}

In this section we mainly focus on very dilute systems for which the classical kinetic theory can serve as a basis of investigations. This regime consists in a dilute assembly of grains interacting through inelastic scattering processes, usually called an inelastic gas. Such a dilute gas of inelastic hard spheres has been intensively studied over the last decade in order to highlight the influence of inelasticity on the properties of a fluid. In this dilute limit, the inelastic Boltzmann equation is usually considered in order to study the grains evolution (11). As compared to the classical Boltzmann equation, the main corrections are due to the inelasticity of collisions, which leads to a timeirreversibility of collisions and to energy dissipation (Fig. 1). These effects are taken into account through the inelasticity parameter $\alpha$, which is defined by the relation:

$$
\left(\boldsymbol{\epsilon} . \mathbf{v}_{i j}\right)^{*}=-\alpha\left(\boldsymbol{\epsilon} \cdot \mathbf{v}_{i j}\right)
$$

where $\mathbf{v}_{i j} \equiv \mathbf{v}_{i}-\mathbf{v}_{j}, \mathbf{r}_{i j} \equiv \mathbf{r}_{i}-\mathbf{r}_{j}$ and $\boldsymbol{\epsilon}$ is a unit vector along the axis

joining the centers of the two colliding hard spheres $i$ and $j$, and $\boldsymbol{\epsilon} \equiv \frac{\mathbf{r}_{i j}}{\left|\mathbf{r}_{i j}\right|}$. The velocities with the ${ }^{*}$ index are the velocities after the collision and the unleashed symbols correspond to the pre-collisional velocities. By definition, 
$\alpha$ belongs to ]0,1], and the elastic limit is recovered for $\alpha=1$.

In (6) , the dependence of $\alpha$ on the relative velocity of the particles has been neglected. This is an approximation since real-world grains have a velocity dependent on $\alpha(12 ; 13)$. One should stress that this simplification has a dramatic but unphysical consequence in MD simulations, the so-called collapse phenomenon (14). This phenomenon, which has been studied in detail during the last decade, is an extreme expression of the clustering instability specific to inelastic fluids and consists in the occurrence of an infinite number of collisions in a finite time.

The dissipativity of collisions contributes to an anomalous exploration of the phase space. Indeed, the transfer of the kinetic energy to the internal degrees of freedom of the grains makes the total kinetic energy of the system to decrease and leads to the irreversibility of the grain dynamics in time. This irreversibility favours some regions in phase space, and generates some complex phenomena, like clustering of trajectories and heterogeneous density regions (Fig. 2). Moreover, it also implies that if no external energy is applied to the system, the system tends toward a perfectly resting state, in which the total kinetic energy vanishes. Consequently, the exploration of the phase space is slowed down. Before the system reaches a complete rest, it passes through different asymptotic states, themselves depending on the initial conditions, because of the lack of ergodicity which develops in the system. Let us stress that this expression of metastability does not rest on equilibrium-like mechanisms. The study of inelastic gases has also led to identifying the main inelastic effects which alter the macroscopic dynamics, namely the anomalous coupling between the local energy and the local density, and the new time scale associated to the dissipative cooling.

One of the macroscopic consequences of inelasticity is the emergence of anomalous transport processes in inelastic gases. This can be found by applying the Chapman-Enskog procedure to the inelastic Boltzmann equation (15), in order to derive hydrodynamic equations for inelastic dilute gases. In doing so, a generalized Fourier law for the heat flux of the granular fluid can be derived:

$$
\mathbf{q}=-\mu \partial_{\mathbf{r}} T-\kappa \partial_{\mathbf{r}} n .
$$

Moreover, the mechanisms which make $\kappa \neq 0$ can be identified. On the one hand, it is a manifestation of the coupling between density and temperature which occurs in granular media. Namely, the local temperatures decrease with different cooling rates depending on the local density. This non-local behaviour discriminates the temperature dependence of neighboring hydrodynamic cells and couples the density field to the heat transport process, leading to an additional term $\sim \partial_{\mathbf{r}} n$, and to a positive definite contribution to $\kappa$. On the other hand, additional contributions to the heat flux come from the shape of the ref- 
erence velocity distribution. This zeroth-order state in the Chapman-Enskog procedure is called the Homogeneous Cooling State, and consists in a state which is and remains homogeneous, and whose time dependence occurs only through the granular temperature. It usually plays the role of the equilibrium state in the statistical description of granular gases. This contribution can be either negative or positive, depending on the variation to the Maxwellian, which is usually measured through the kurtosis of the velocity distribution.

A striking consequence of this generalized Fourier law is the phenomenon of temperature inversion in vibrated granular systems. This phenomenon occurs in systems subject to gravitation and to which energy is supplied by a vibrating bottom wall. Because of the energy injection, the inelastic fluid attains an asymptotic stationary distribution which is different from the total rest state, and whose temperature is non-vanishing. Several authors have shown that the temperature profile is not uniform in the system $(20 ; 21 ; 22)$. Moreover, the granular temperature has been shown to exhibit a minimum at some distance from the boundary. This fact, which implies that the granular temperature can increase with increasing height, has been predicted using Eq. (2), and verified by experiments and numerical simulations (하). A question remains on whether the Fick and Ohm equations also have to be appropriately modified in their own context. Another question pertains to Onsager relations validity, and whether they should be generalized.

The non-Maxwellian features of velocity distributions arising in inelastic gases are also an interesting phenomenon. Indeed, their tendency toward overpopulated high energy tails is non trivial, and the specific shape of the tail depends on the details of the model. However, overpopulation seems to be a generic feature of inelastic gases (11). This property has been predicted theoretically and numerically, and observed experimentally in a large number of situations, see (6). From a theoretical point of view, simplified kinetic models have been introduced to study the formation of these fat tails, in pure systems and in mixtures (16). These models, which rest on a mathematical simplification of the collision operator in the inelastic Boltzmann equation, are usually called Maxwell Models. Usually, two kinds of asymptotic velocity distributions are considered: (i) scaling solutions, which occur when the grains evolve freely, without external forcing and correspond to a Homogeneous Cooling State, (ii) heated stationary solutions, which are obtained by injecting energy into the system to counterbalance the energy loss. The energy is usually introduced by stochastic forces, usually of Langevin type. This mimics properties of the vibro-fluidized granular media. In (17; 18), another class of asymptotic solutions has been studied, namely stationary solutions of the unforced case. By using similarities between the Maxwell model and a random walk in velocity space, it is shown theoretically and numerically that almost stationary solutions exist. This means that the core of these distributions is stationary for an arbitrary time extent, while the total energy in the system decreases expo- 
nentially fast. It can be shown that these solutions correspond to truncated Lévy distributions.

Finally, let us recall the granular Demon experiment, conceived to visualize the energy-density coupling characteristic of granular matter. It consists of a box divided into two equal compartments by a vertical wall starting from the bottom of the box, in which a hole allows the passage of the grains from one compartment to the other. The box is filled with inelastic identical particles submitted to gravitation. Energy is supplied by a vibrating bottom wall. This simple system can be shown to exhibit an order-disorder transition. Indeed, for a high energy input, the system presents a homogeneous steady state, while when the energy input is decreased, a phase transition occurs and an asymmetric steady state prevails. The effusive model proposed by Eggers (23) explains this transition. In (19), this system has been studied by using a stochastic urn model of Lipowski, see also (5; 24). Several generalizations of the original Maxwell Demon experiment have been considered, and have led to a rich phenomenology: (i) systems composed by an arbitrary number of compartments, leading to metastable states. (ii) systems where energy is input asymmetrically in the urns, showing hysteresis and strong similarities to the behavior of a ferromagnetic system in a external magnetic field. (iii) the original experiment applied to mixtures, leading to horizontal segregation and the emergence of non-stationary oscillating behaviors, called the granular clock.

\section{Ballistic Magnetic Deposition Model}

At higher density, granular media lock into piles. In constructing and describing granular piles, it is crucial to remember that basic entities are not made of symmetrical units, like hard spheres; even if they can be thought to be made of hard cores (25), like rice grains or sand grains (26; 27), they can be hardly assumed to be spherical or disk shaped and perfectly sliding on each other. Specific angles of repose (28), jams and arches (29) are thought to originate from these contact forces.

We have introduced a so called magnetically-controlled ballistic rain-like deposition (MBD) model (7; 30; 31) of granular piles and numerically investigated its static properties in 2D. The grains are assumed to be elongated disks characterized by a two-state scalar degree of freedom, called the "nip". The direction of a nip can represent e.g. anisotropy of grain, or the position of a grain with respect to neighboring entities. The nip-nip interaction is described through the well known Ising-like Hamiltonian. An external field of arbitrary origin can be imagined to forcefully allow the grain to rotate during its deposition. This effect is introduced through a parameter $q$. 
The simulation algorithm creates a pile under a fixed probability $q$ for grain rotation, or nip change of sign, during the ballistic grain fall; the probability to choose the "up" direction (or +1 value) is $q$.

The local energy gain

$$
E=-J \sum_{<i, j>} n_{i} n_{j}
$$

is calculated at each time step since $n_{j}$ can take the value +1 or -1 depending on whether the grain long direction is either vertical or horizontal.

If the "energy gain" $\Delta E$ is negative the grain sticks to the cluster immediately in its "nip" state. In the opposite case $(\Delta E>0)$ the grain sticks to the cluster with a probability $\exp (-\Delta E)$ where $\Delta E$ is the local gain in the energy. If the grain does not stick it continues to fall down. Of course, if the site just below the grain is occupied, the grain immediately stops and sticks to the cluster.

The density and the "niptization" (the order parameter for the grain orientation) have been measured, as well as the fractal-like characteristics of clusters. It is of interest to report in the present context that clusters of various sizes, with specific size distributions, occur as a function of $q$ and $J$ (Figs.3-4). It has been noticed that the higher the nip-nip interaction strength, the bigger the difference between the various piles $(30 ; 31)$.

It seems (31) that one can distinguish pile growth conditions and pile structure depending on whether $q<q_{c}(J)$ or $q>q_{c}(J)$. Two different cluster-mass regimes have been identified, through the cluster-mass distribution function. It can be exponential or follow a power law form depending on whether the nip flip (or grain rotation) probability $q$ is small or large. It is thought that the regimes are distinguishable because of a percolation-like transition at finite $q_{c}$, being $q_{c} \simeq 0.85$ and $q_{c} \simeq 0.75$, respectively for the $J<0$ and $J>0$ cases respectively. There is no theory for such features at the moment.

The practical interest is then to calculate the stress net in the pile. This is a hyperstatic problem (32). However we have observed (33) that for hard spheres the most frequent contact number is 4 . Therefore, in the case of elongated disks the stress net for the BMD model might be more easily handled or solved in two dimensions (2D) than in $3 \mathrm{D}$. The net has been found to be being unique in 2D. Moreover this contact number value seems to remove constraints related to rotation-frustration problems.

Extensions toward binary, polydispersed or more complex objects have not received much attention. Yet recent studies concerning granular piles suggest the interest of such further investigations (34). 


\section{Hybrid Sand Pile Model}

SOC phenomena have recently attracted much interest. Their most intriguing property reside in the 'spontaneous' formation of scale-free spatio-temporal patterns with power-law correlations between various quantities. On the one hand these correlations closely resemble those appearing at critical points in continuous phase transitions; on the other hand, while the critical state at such phase transitions is temperature- or external-field-driven, a SOC state is believed to form spontaneously, without fine-tuning of any external parameters. The phenomenon basic understanding is still open and the satisfactory definition of SOC phenomena remains elusive. It was thus of interest to test the robustness of SOC introducing Hamiltonian like constraints. Whence we have numerically investigated a hybrid model, i.e. using the Blume-Emery-Griffiths (BEG) (10) model for the Hamiltonian part,

$$
H=-J \sum_{\langle i, j\rangle} S_{i} S_{j}-K \sum_{\langle i, j\rangle} S_{i}^{2} S_{j}^{2}+D \sum_{i} S_{i}^{2},
$$

and the Bak-Tang-Wiesenfeld sand pile model (9) for the SOC part. The Blume-Emery-Griffiths (BEG) model is a mere generalization of the above nip model into spin space. We have considered a two-dimensional honeycomb lattice of linear size $L$ (Fig.5) . Each lattice node $j$ can be interpreted either as the number of sand grains $h_{j} \in\{0,1,2\}$ on a site or a spin variable $s_{j} \in$ $\{-1,0,1\}$. Of 6 mappings of heights $h_{j}$ on spins $s_{j}$, we choose the one in which $h_{j}=0,1,2$ corresponds to $s_{j}=0,-1,+1$, respectively.

A node where $h_{j}>2$ is called 'active', such that 3 grains are transferred from $j$ to its 3 nearest neighbors, one grain for each neighbor. The process can spontaneously continue, giving rise to an avalanche of a priori unknown size. The whole procedure of adding a grain at a randomly chosen node and then completely relaxing, i.e. the resulting avalanche, can be treated as a Markov process on lattice stable configurations. It is clear that the set of allowed height (or spin) configurations is restricted to the set of the recurrent states of this Markov process, i.e. only the stable lattice configurations that can be reached arbitrarily many times are considered in this sand pile 'add-and-relax' process.

Sand pile models are known to exhibit strong finite-size effects (35). This reflects the fact that the system is not uniform - the average density on the boundary is different than that in the bulk. For the sand pile part of this hybrid model we have used open boundary conditions (BC). However, for the Hamiltonian component, in order to minimize finite-size effects, we have adopted periodic BC in our simulations. 
Since the phase space of our model is limited to recurrent states of a Markov process, there is no doubt that the system is theoretically ergodic. However, transitions between some states can be so infrequent that they will practically never occur in computer simulations. This problem can be particularly serious for transitions related to large avalanches. Any breaking of ergodicity, often related to 'glassyness' of a system, manifests itself in computer simulations through anomalously slow relaxation. This has been observed in our simulations.

Nevertheless we have found interesting features. First, the high-temperature properties of this hybrid SOC model are very unusual: while in standard Hamiltonian systems the magnetisation disappears at high temperatures, a nonzero, positive magnetisation $m$ occurs for infinite $T$ for any values of the control parameters $J, K$, and $D$.

It can be argued (36) that only two magnetic phases can exist: one ferromagnetic $(F)$ and one antiferromagnetic $(A)$ one. Note that this reduction of the number of possible phases is a consequence of the constraints imposed by the sand pile subsystem, and has nothing to do with the BEG criticality.

The temperature dependence of the specific heat has been calculated for different lattice sizes from energy fluctuations,

$$
C=\frac{N}{T^{2}}\left(\left\langle u^{2}\right\rangle-\langle u\rangle^{2}\right)
$$

It has been found that a maximum develops in the specific heat near $T=1.55$, while $C$ decays as $T^{-2}$ at high temperatures. Moreover very slow relaxation occurs near this temperature, as for a second order phase transition, allowing us to call it $T_{c}$. Our results suggest that the effect of slow relaxation dynamics is amplified by nonlocal constraints of the sand pile model.

One signature of 'criticality' of the BTW sand pile model is a power-law decay of two-point correlation functions $P_{k l}(r)$, of nodes at a distance $r$ apart, having heights $k$ and $l(0 \leq k, l \leq 3)$. Let $P_{k}$ be the probability that a given site has $k$ grains. It was proved that in a two-dimensional sand pile

$$
P_{k l}(r)=P_{k} P_{l}+p_{k l} r^{-4}+\ldots
$$

for $k=l=0$ in the bulk (37) and for all $0 \leq k, l \leq 3$ near the boundary of the system (38).

We have examined the case $k=l=0$. The results for a rather high temperature $T=10$, where the BEG interactions should not play a significant role, are presented in Fig. 6. As can be seen, an exponential fit is far better than 
an algebraic one. This suggests that the 'criticality' of the sand pile model is lost, and the correlations are dominated by the Hamiltonian subsystem. We believe that this conclusion remains valid for all finite temperatures.

Conversely we find that the criticality of the BEG model is not affected by the criticality of the BTW model. However, even weak interactions of the BEG model destroy self-organized criticality of the BTW model.

In the above study, the lattice basis is a regular structure. If the basis has some fractal structure it has been shown elsewhere that the avalanches present an extra feature, a log-periodicity, depending on the fractal dimension and connectivity of the lattice (27). Translated into a complex fractal dimension (39) this sandpile model has then served as an analogy for describing endogenous financial crashes (40).

\section{Conducting Densely Packed Matter}

There is to our knowledge very few papers dealing with the temperature dependence of electrically conductive packed materials. The electrical properties of such systems are of interest because there is often a strong modification of the interface, when an electric current is imposed, - leading to a strong modification of the oxide layers usually formed at the grain surfaces (41; 42), even up to welding.

Such systems are usually thought to behave like a disordered resistor network characterized by electronic conduction occurring in a strongly localized regime. Whence transport properties depend whether the system is above a percolation transition or not. Carrier hopping between grains dominate the resistance behavior. Whence such systems display certain features of the variable-rangehopping (VRH) phenomenon type observed in doped semiconductors. In particular, the electrical resistivity of such systems has been observed to obey a so called "fractional temperature dependence",

$$
\rho(T)=\rho_{0} \exp \left[\left(\frac{T_{0}}{T}\right)^{p}\right]
$$

where $T$ is the temperature, $T_{0}$ a characteristic temperature, and $p=1 /(d+1)$, in terms of $d$ the dimensionality of the system (43; 44; 45).

It might be that by avoiding the oxide layer the electrical conduction mechanism will also probe the internal structure of the grain, but the more so the intergrain contact might not conform to the VRH mechanism. 
Therefore we have synthesized and compacted a crystalline granular metallic system,i.e. $\mathrm{CaAl}_{2}$. We have used an original route (46) and have obtained tiny crystals. The chemical analysis has indicated a complex microstructure inherent to the phase diagram intricacies (47). An EDX analysis indicates that the system is made of dendrites embedded in a matrix. The dendrites are $A l$ rich and made of $\mathrm{CaAl}_{2}$ while the matrix is $A l$ poor and has a composition close to $C a_{0.6} A l_{0.4}$, - in fact a mixture of $C a_{13} A l_{14}$ and $C a_{8} A l_{3}$ according to the phase diagram. These phases have already been studied (48) and have been found to be metallic with a very similar electrical resistance.

The electrical resistivity and the thermoelectric power of the packed crystal have been measured under standard conditions. Both properties show three regimes as a function of temperature (Figs. 7-8). It should be pointed out first that the electrical resistivity continuously decreases between 15 and 235 $\mathrm{K}$ with various dependences. The electrical conduction process is found to have a temperature behavior $\rho(T) \simeq T^{-3 / 4}$ at low temperature (Fig.7). This is best interpreted in terms of a thermal effect, on a resistive (geometrically disordered) backbone, not changing with temperature, - in this low $T$ range. The $60-70 \mathrm{~K}$ break (or crossover) indicates the energy range at which the thermal process takes over on the geometric disorder. At higher temperature, the smooth decay of $R(T)$ with increasing $T$ can be attributed to the FermiDirac function behavior increasing width as $T$ increases and further charge carrier delocalization.

The (positive) thermoelectric power (46) shows a bump near 60K, after an unusual square root of temperature dependence at low temperature (Fig.8). Above $100 \mathrm{~K}$, a log-log plot indicates that $Q(T)$ behaves like $T^{3 / 4}$, thus not quite linearly as it should be for metallic systems at high $T$. Recall that since a TEP measurement implies no external electrical current. it is unlikely that some "barrier ageing" or "hot spots" take place in the temperature range of interest. Within our line of thought, both increase in charge and heat transport as a function of temperature and the large thermoelectric effect at room temperature can thus be understood as resulting from a delocalization process on the intricate barrier network, with competing characteristic mean free paths, and a weakening of the contact TEP due to Fermi surface widening with temperature.

Therefore the three observed regimes are thought to indicate a competition between geometric and thermal processes in weakly conducting clusters of densely packed granular matter. More work is surely to be done in order to verify the above observed behaviors in other cases and to relate the macroscopic transport properties to the internal states of the system. 


\section{Conclusions}

Granular systems have been considered as a part of applied physics and technology. Except for some notable attempts by Faraday and Coulomb, they have not reached the attention of the physics community until recently.

There are several reasons for this new interest. A first reason arises from the apparent simplicity of granular matter, which surprisingly leads to a very rich and often counterintuitive behavior. Entropy considerations may be outweighted by dynamical effects while the exploration of phase space is unusual rending the system non-ergodic. The dissipativity of collisions contributes to this anomalous exploration of phase space. Indeed, the loss of kinetic energy causes a decrease of the total energy of the system, and leads to irreversibility of the grains dynamics. The simplicity of granular materials originates from the contact forces acting between their components.

We have first focused on dilute systems, so that the methods of kinetic theory may be generalized. In this regime, a dilute assembly of inelastic grains, usually called an inelastic gas, has been much studied over the last decade in order to highlight the influence of inelasticity on the properties of a fluid. Amongst others, it has been used in order to provide a kinetic foundation to hydrodynamic-like equations for these systems. We have recalled their characteristic tendency to clustering. The study of inelastic gases has also led to an identification of the main inelastic effects which alter the macroscopic dynamics, namely the coupling between the local energy and the local density in these systems, a new term in Fourier equation of heat conduction, and a new time scale associated to the dissipative cooling.

Next we have outlined a model of gravity deposition for anisotropic 2D gains, in a more simple way: the gains can either be with their long axis vertical or horizontal. We have observed that according to the probability of one orientation being more favorable and with a condition of sticking based on an Ising like Hamiltonian different types of clusters emerge, - distributed in different ways around a possible percolation like transition.

Next we have observed the interplay of SOC and Hamiltonian constraints. Finally we have recalled anomalous temperature behaviors of dense granular electrically conducting clusters.

\section{Acknowledgments}

MA and KT thank the organizers of the 41th Karpacz school conference for their welcome, stimulating discussions and comments. MA, RL and KT thank also ARC 02-07/2093 for financial support. MA and MP acknowledge financial 
support from NATO and CGRI-KBN bilateral exchange agreements. Paulette CLIPPE and Andrzej PEKALSKI should be much thanked.

\section{References}

[1] P. G. de Gennes, Superconductivity in Metals and Alloys, Benjamin, New York, 1966.

[2] P. Bak, How Nature Works, Oxford Univ. Press, New York, 1997.

[3] H. M. Jaeger, Chu-heng Liu, S. R. Nagel, Phys. Rev. Lett. 62 (1989) 40.

[4] J.B. Knight, C. G. Fandrich, C. N. Lau, H. M. Jaeger, S. R. Nagel Phys. Rev. E 51 (1995) 3957.

[5] A. Lipowski, these proceedings.

[6] R. Lambiotte, Ph. D. thesis, Univ. Libre de Bruxelles, (2004) unpublished.

[7] K. Trojan, M. Ausloos, Physica A 326 (2003) 491.

[8] E. Caglioti, V. Loreto, H. J. Herrmann, M. Nicodemi, Phys. Rev. Lett. 79 (1997) 1575 .

[9] P. Bak, C. Tang, K. Wiesenfeld, Phys. Rev. Lett. 59 (2003) 381.

[10] M. Blume, V.J. Emery, R.B. Griffiths, Phys. Rev. A 4 (1971) 1071.

[11] M. H. Ernst, R. Brito, J. Stat. Phys. 109 (2002) 407.

[12] R. Ramirez, T. Poschel, N. V. Brilliantov, T. Schwager Phys. Rev. E 60 (1999) 4465.

[13] N. V. Brilliantov, T. Poschel, Phys. Rev. E 61 (2000) 5573.

[14] S. McNamara, W. R. Young, Phys. Rev. E 50 (1994) R28.

[15] J. W. Dufty, Adv. Compl. Syst. 4 (2001) 397.

[16] R. Lambiotte and L. Brenig, Phys. Rev. E, in press; cond-mat/0508768

[17] R. Lambiotte and L. Brenig, Phys. Lett. A 345 (2005) 309; cond-mat/0507573

[18] R. Lambiotte, L. Brenig and J.M. Salazar, Physica A, in press; cond-mat $/ 0510108$

[19] R. Lambiotte, J.M. Salazar and L. Brenig, Phys. Lett. A 343 (2005) 224; cond-mat/0507478

[20] R. Soto, M. Mareschal, D. Risso, Phys. Rev. Lett. 83 (1999) 5003.

[21] J. Brey, D. Cubero, F. Moreno, M. J. Ruiz-Montero, Europhys. Lett. 53 (2001) 432.

[22] B. Meerson, T. Poschel, Y. Bromberg, Phys. Rev. Lett. 91 (2003) 024301.

[23] J. Eggers, Phys. Rev. Lett. 83 (1999) 5322.

[24] A. Lipowski, M. Droz, Phys. Rev. E 65 (2002) 031307.

[25] H. A. Makse, J. Brujic, S. F. Edwards, in The Physics of Granular Media, eds. H. Hinrichsen and D.E. Wolf, Wiley-VCH, Weinheim, 2004.

[26] S. R. Nagel, Rev. Mod. Phys. 64 (1992) 321.

[27] N. Vandewalle, R. D'hulst, M. Ausloos, Phys. Rev. E 59 (1999) 631.

[28] H. J. Herrmann, in Physics of dry granular media, eds. H. J. Herrmann, J.-P. Hovi, S. Luding, Kluwer, Dordrecht, 1998. 
[29] S. Luding, T.A.S.K. Quarterly, Scientific Bulletin of Academic Computer Centre of the Technical University of Gdansk, 2 (1998) 417.

[30] K. Trojan, M. Ausloos, Phys. Rev. E 69 (2004) 052301.

[31] K. Trojan, M. Ausloos, R. Cloots, Int. J. Mod. Phys. C 15 (2004) 1121.

[32] J. H. Snoeijer, T. J. H. Vlugt, W. G. Ellenbroek, M. van Hecke, J. M. J. van Leeuwen, Phys. Rev. E 70 (2004) 061306.

[33] K. Trojan, M. Ausloos, unpublished.

[34] H. J. Herrmann, Physica A 191 (1992) 263.

[35] S. Lubeck, Phys. Rev. E 56 (1997) 5138.

[36] Z. Koza, M. Ausloos, unpublished.

[37] S. N. Majumdar, D. Dhar, J. Phys. A 24 (1991) L357.

[38] E. V. Ivashkevich, Phys. Rev. Lett. 76 (1996) 3368.

[39] D. Sornette, Phys. Rep. 378 (2003) 1.

[40] M. Ausloos, K. Ivanova, N. Vandewalle, in Empirical sciences of financial fluctuations. The advent of econophysics, H. Takayasu, Ed. ,Springer Verlag, Berlin, 2002, pp. 62-76.

[41] S. Dorbolo, M. Ausloos, N. Vandewalle, Appl. Phys. Lett. 81 (2002) 936.

[42] S. Dorbolo, M. Ausloos, N. Vandewalle, Eur. Phys. J. B 34 (2003) 201.

[43] N. F. Mott, E. A. Davies, Electron Processes in NonCrystalline Materials, Clarendon, Oxford, 1979.

[44] N. F. Mott, Metal-Insulator Transitions, Taylor \& Francis, London, 1990.

[45] B. I. Shklovskii, A. I. Efros, Electronic Properties of Doped Semiconductors, Springer, Berlin, 1984.

[46] M. Ausloos, M. Pekala, J. Latuch, J. Mucha, Ph. Vanderbemden, R. Cloots, J. Appl. Phys. 96 (2004) 7338.

[47] K. Ozturk, Long-Qing Chen, Zi-Kui Liu, J. Alloy Comp. 340 (2002) 199.

[48] B. Huang, J. D. Corbett, Inorg. Chem. 37 (1998) 5827. 

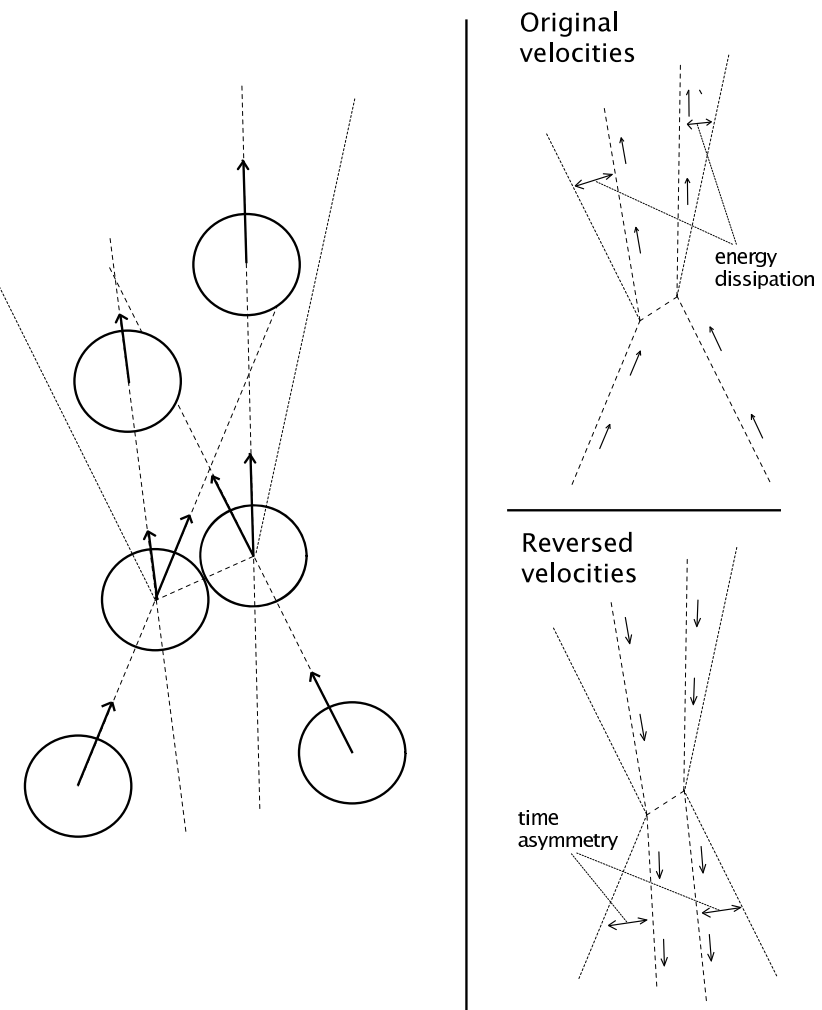

Fig. 1. Collision between inelastic hard spheres. Inelasticity makes the post-collisional velocities more parallel as compared to an elastic collision. This is a direct effect of inelasticity which lowers the quantity $\left(\boldsymbol{\epsilon} \cdot \mathbf{v}_{12}\right)$ during one collision. Figures on the right illustrate the irreversibility of the microscopic dynamics: the inverted trajectories are no longer the same as the direct ones

\section{Figure Captions}



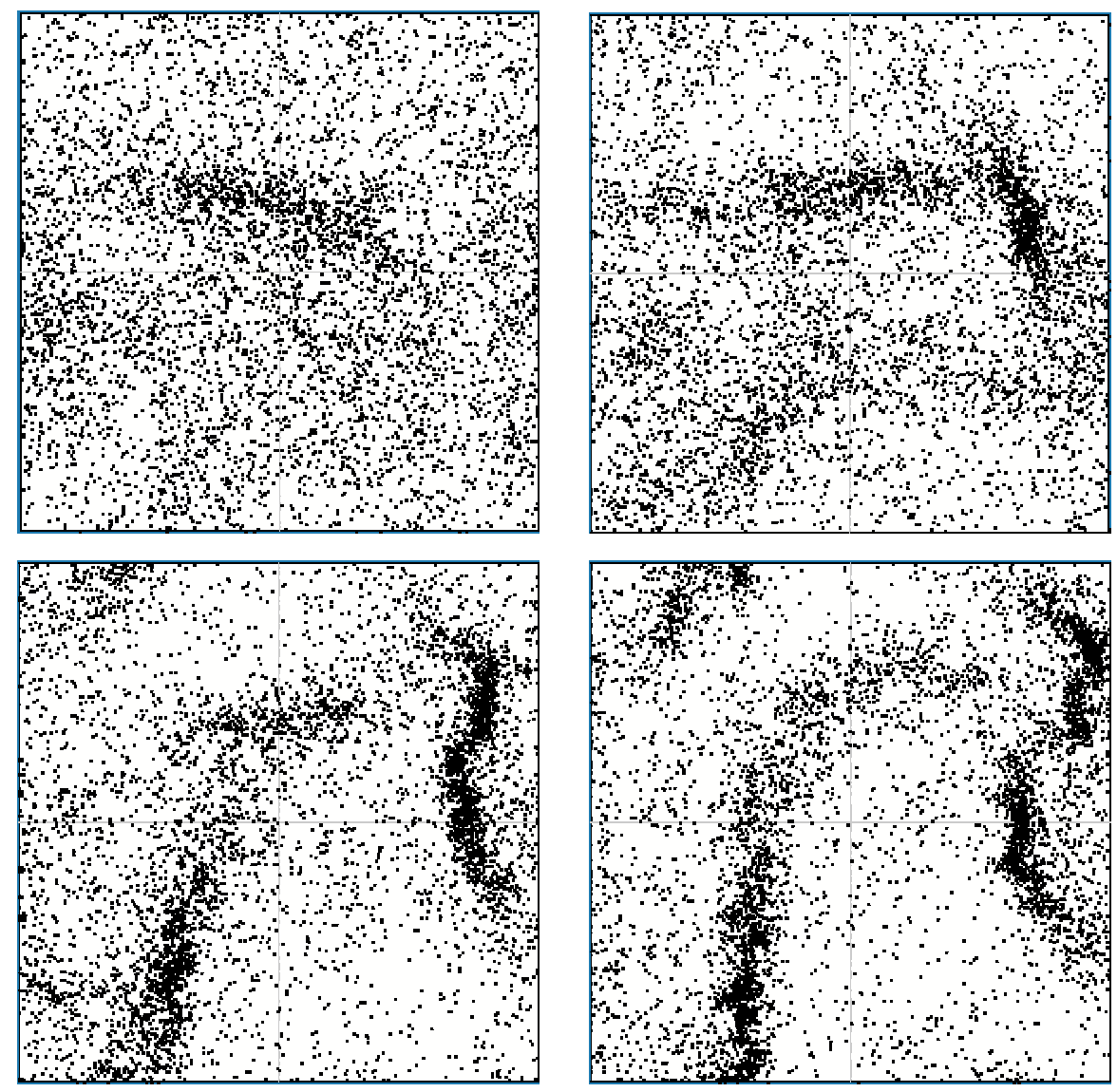

Fig. 2. Aggregation tendency and emergence of clustering in a freely cooling granular gas. The system is composed of 5000 inelastic discs and $\alpha=0.9$ in Eq.(1)
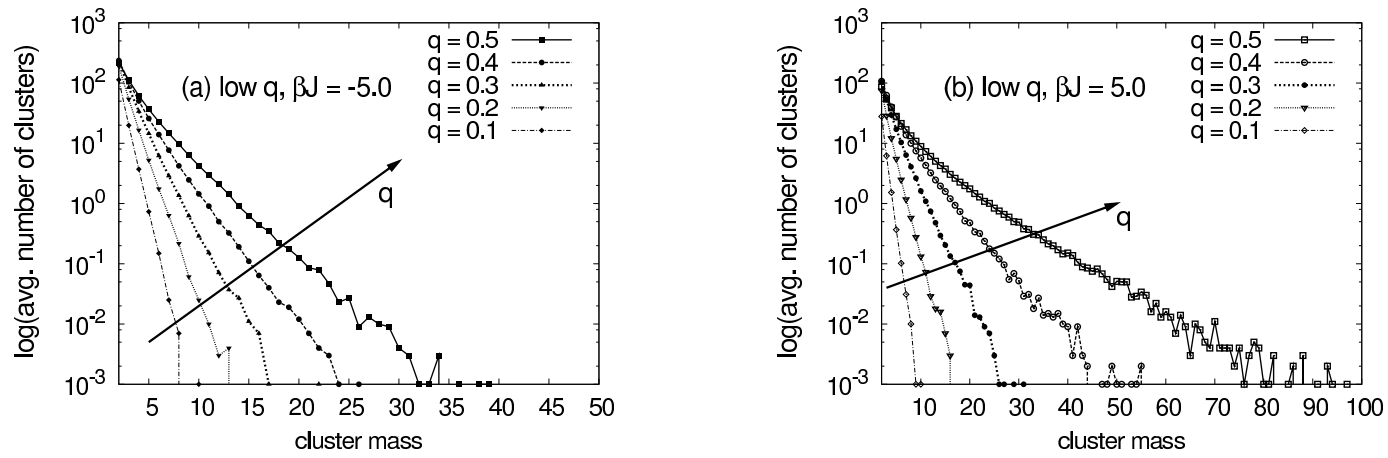

Fig. 3. Semilog plots of the cluster size distribution for low $q$ values: (a) $\beta J=-5$, (b) $\beta J=5$. Observe the exponential law-like behavior of the distribution 

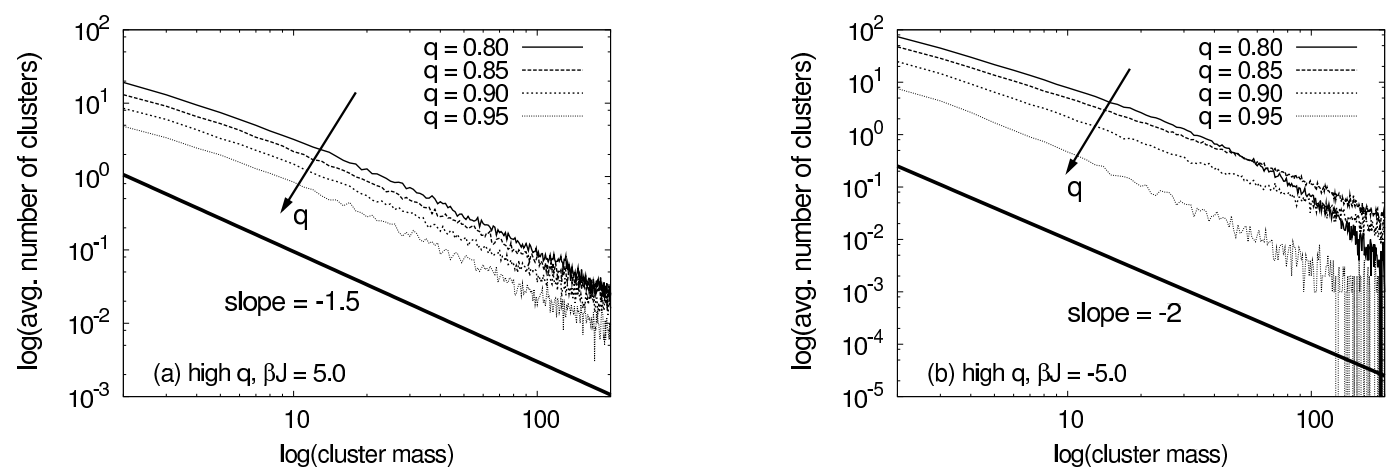

Fig. 4. Log-log plots of the cluster size distribution for high $q$ values: (a) $\beta J=-5$, (b) $\beta J=5$. Notice the power law-like behavior of the distribution

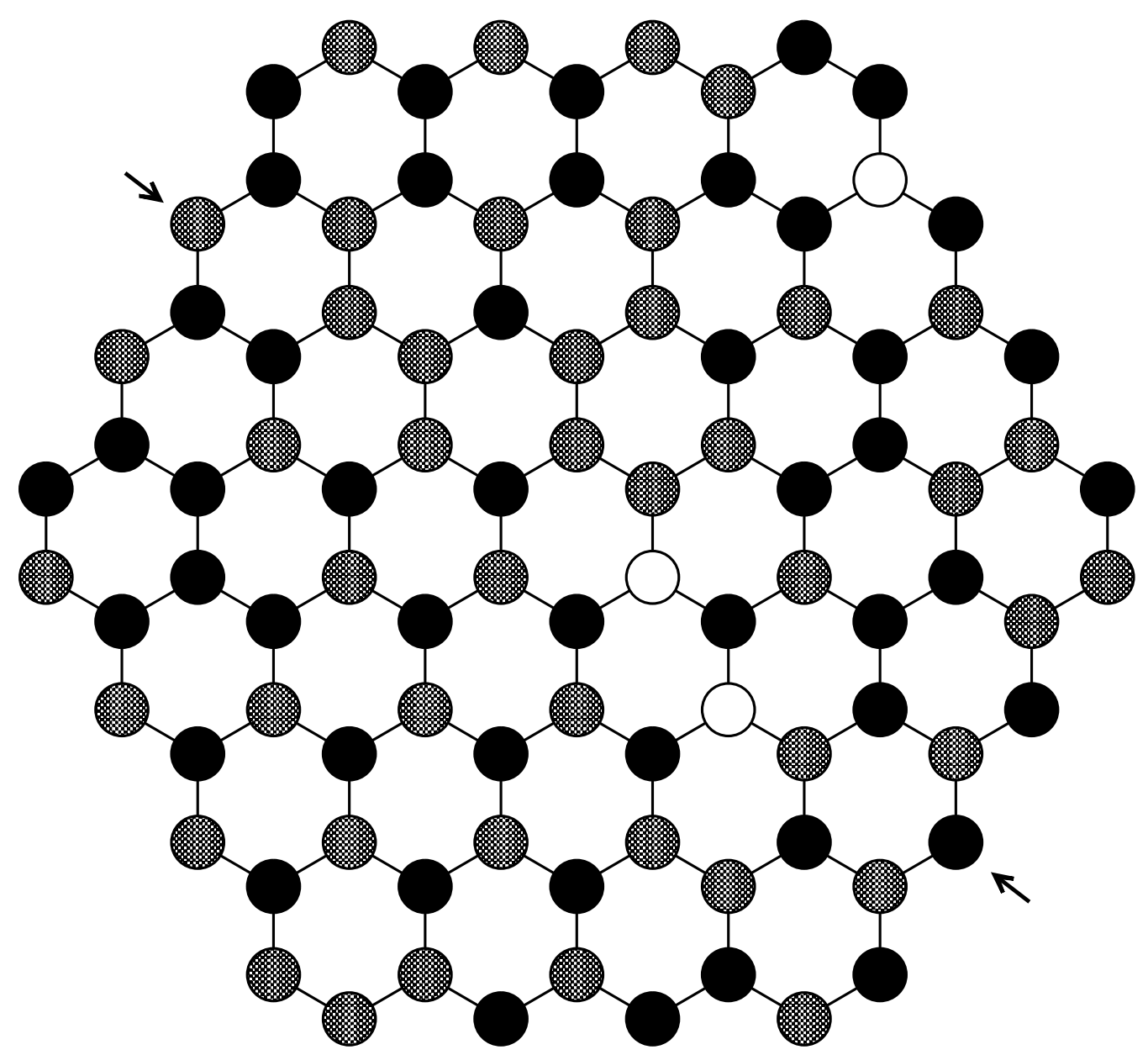

Fig. 5. An example of an allowed configuration on a honeycomb lattice of linear size $L=4$. Empty, shadowed, and filled circles represent the nodes with heights $h_{j}=0,1,2$ (or spins $s_{j}=0,-1,+1$ ), respectively. The arrows show an example of a pair of boundary nodes that interact via Hamiltonian (3). 


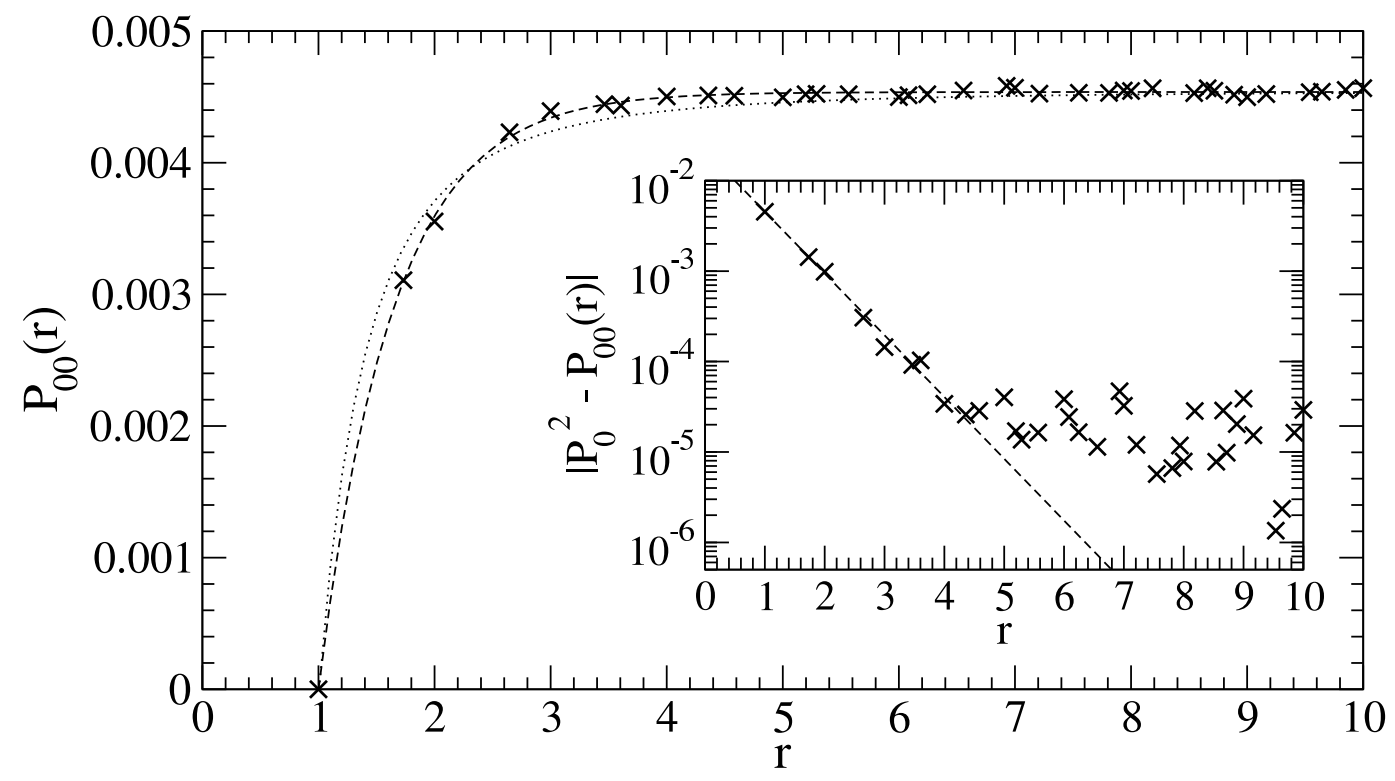

Fig. 6. Probability $P_{00}(r)$ of finding two empty lattice nodes $r$ units apart $(r=1$ corresponds to the distance between nearest neighbor nodes). The data were obtained for $L=40$ and $T=10$. The dashed line is an exponential fit (of the form $\left.a_{0}+a_{1} \exp \left(-a_{2} r\right)\right)$, and the dotted line presents a power-law fit $\left(a_{0}+a_{1} r^{-a_{2}}\right)$. The inset presents the semi-log plot of $\left(P_{0}\right)^{2}-P_{00}(r)$ and shows that exponential fit is based on 9 points and includes 2 decades on the ordinate axis. 


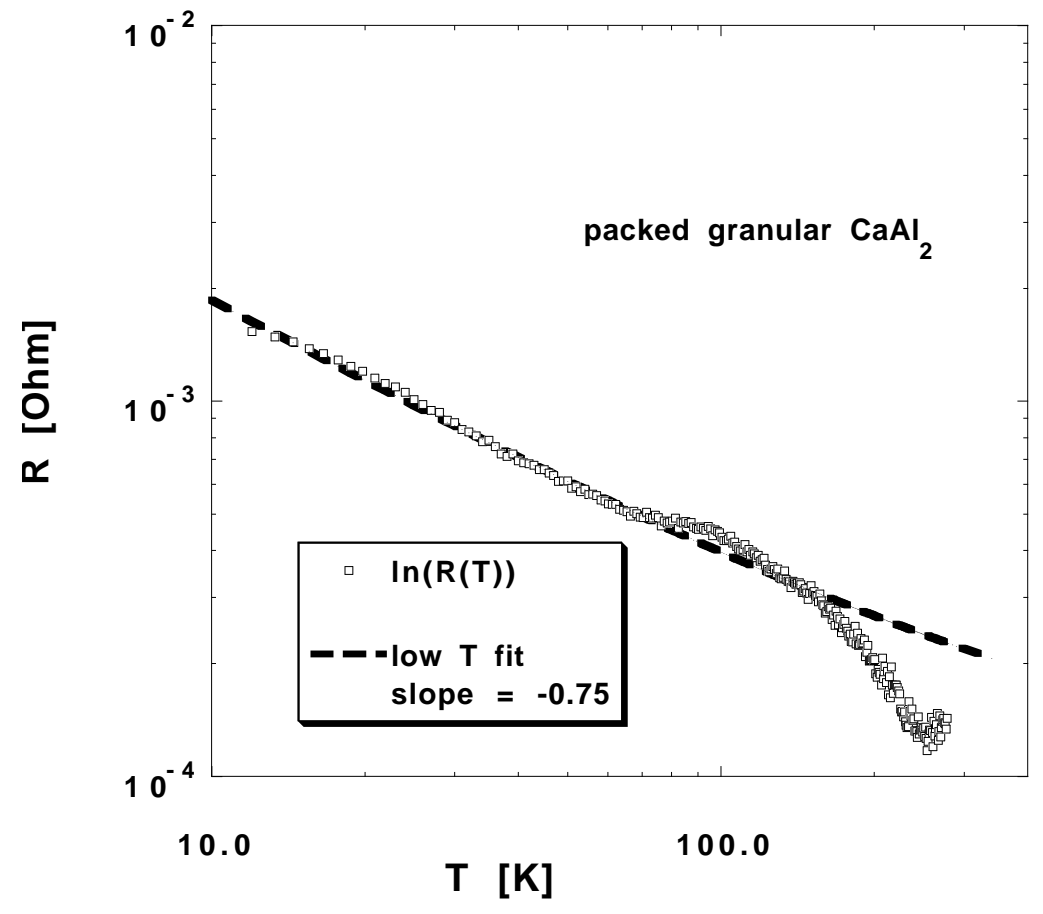

Fig. 7. Electrical resistance $R$ vs. temperature $T$ of a densely packed granular $\mathrm{CaAl}_{2}$ on a log-log plot, with a fit to a line with slope $=-3 / 4$ at low temperature 


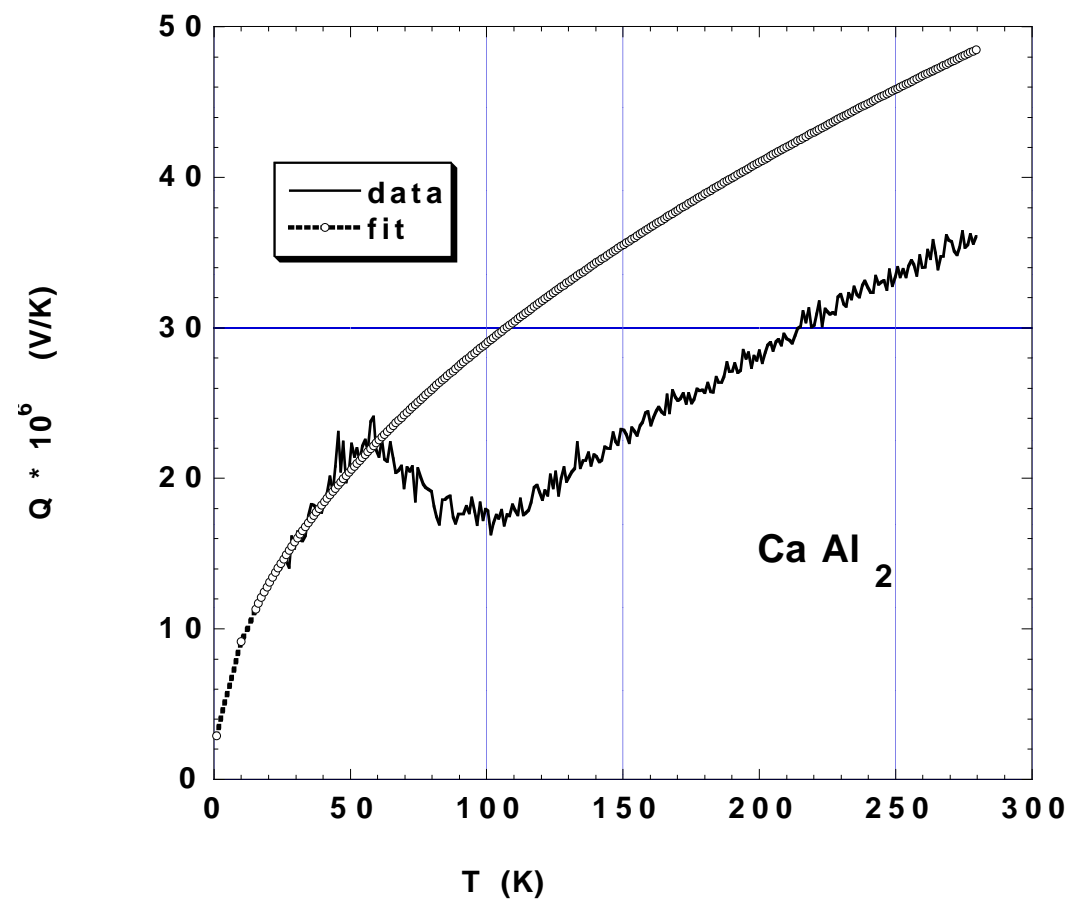

Fig. 8. Thermoelectric power $Q(T)$ vs. temperature $T$ of a densely packed granular $\mathrm{CaAl}_{2}$ with a square root fit at low temperature 